\title{
Measuring the Spins of Accreting Black Holes
}

\author{
Jeffrey E. McClintock ${ }^{1}$, Ramesh Narayan ${ }^{1}$, Shane W. Davis ${ }^{2}$, Lijun Gou ${ }^{1}$, Akshay \\ Kulkarni $^{1}$, Jerome A. Orosz ${ }^{3}$, Robert F. Penna ${ }^{1}$, Ronald A. Remillard ${ }^{4}$, James F. Steiner ${ }^{1}$
}

\begin{abstract}
A typical galaxy is thought to contain tens of millions of stellar-mass black holes, the collapsed remnants of once massive stars, and a single nuclear supermassive black hole. Both classes of black holes accrete gas from their environments. The accreting gas forms a flattened orbiting structure known as an accretion disk. During the past several years, it has become possible to obtain measurements of the spins of the two classes of black holes by modeling the X-ray emission from their accretion disks. Two methods are employed, both of which depend upon identifying the inner radius of the accretion disk with the innermost stable circular orbit (ISCO), whose radius depends only on the mass and spin of the black hole. In the $\mathrm{Fe} \mathrm{K} \alpha$ method, which applies to both classes of black holes, one models the profile of the relativistically-broadened iron line with a special focus on the gravitationally redshifted red wing of the line. In the continuumfitting method, which has so far only been applied to stellar-mass black holes, one models the thermal X-ray continuum spectrum of the accretion disk. We discuss both methods, with a strong emphasis on the continuum-fitting method and its application to stellar-mass black holes. Spin results for eight stellar-mass black holes are summarized. These data are used to argue that the high spins of at least some of these black holes are natal, and that the presence or absence of relativistic jets in accreting black holes is not entirely determined by the spin of the black hole.
\end{abstract}

\section{Introduction}

Our focus throughout is on the two principal methods for measuring the spins of accreting black holes: modeling the thermal continuum X-ray spectrum and modeling the profile of

\footnotetext{
${ }^{1}$ Harvard-Smithsonian Center for Astrophysics, 60 Garden Street, Cambridge, MA 02138

${ }^{2}$ Canadian Institute for Theoretical Astrophysics, Toronto, ON M5S3H4, Canada

${ }^{3}$ Department of Astronomy, San Diego State University, 5500 Companile Drive, San Diego, CA 92182

${ }^{4}$ MIT Kavli Institute for Astrophysics and Space Research, MIT, 70 Vassar Street, Cambridge, MA 02139
} 
the relativistically-broadened $\mathrm{Fe} \mathrm{K} \alpha$ line. The continuum-fitting ( $\mathrm{CF}$ ) method, has thus far only been applied to stellar-mass black holes in X-ray binaries, whereas the Fe $\mathrm{K} \alpha$ method has been applied to both stellar-mass $\left(M \sim 10 M_{\odot}\right)$ and supermassive $\left(M \sim 10^{6}-10^{10} M_{\odot}\right)$ black holes 1 . This paper is chiefly focused on measuring the spins of stellar-mass black holes via the $\mathrm{CF}$ method because we have been deeply engaged in this work during the past several years. In Section 6, we secondarily discuss the Fe $\mathrm{K} \alpha$ method, which is very important because it is the primary approach to measuring the spins of supermassive black holes.

We note that spin estimates have also been obtained by modeling the high-frequency X-ray oscillations (100-450 Hz) observed for several stellar-mass black holes (Wagoner et al. 2001; Török et al. 2005). At present, this method is not providing dependable results because the correct model of these oscillations is not known. X-ray polarimetry is another potential avenue for measuring spin (Dovčiak et al. 2008; Li et al. 2009; Schmoll et al. 2009), which may be realized soon with the 2014 launch of the Gravity and Extreme Magnetism Small Explorer (Swank et al. 2008). Meanwhile, several other methods of measuring spin have been proposed or applied (e.g., Takahashi 2004; Barai et al. 2004; Huang et al. 2007; Suleimanov et al. 2008; Shcherbakov \& Huang 2011).

\section{Stellar-Mass Black Holes in X-ray Binaries}

Observations in 1972 of the X-ray binary Cygnus X-1 provided the first strong evidence that black holes exist. Today, a total of 23 such X-ray binary systems are known that contain a compact object too massive to be a neutron star or a degenerate star of any kind (i.e., $M>3 M_{\odot} ;$ Özel et al. 2010). These compact objects, which have typical masses of $10 M_{\odot}$, are referred to as black holes. Their host systems are mass-exchange binaries containing a nondegenerate star that supplies gas to the black hole via a stellar wind or via Rochelobe overflow in a stream that emanates from the inner Lagrangian point. The mass-donor star in the Roche-lobe overflow systems is typically a low mass $\left(M \sim 1 M_{\odot}\right)$ sun-like star, and the X-ray source is transient, alternating between yearlong outbursts $\left(L_{\max } \sim L_{\text {Edd }}=\right.$ $\left.1.3 \times 10^{39} M / 10 M_{\odot} \operatorname{erg~s}^{-1}\right)$ and years or decades of quiescence $\left.\left(L \sim 10^{-7} L_{\mathrm{Edd}}\right)\right)^{2}$. The windfed X-ray sources, on the other hand, are fueled by massive hot stars $\left(M \gtrsim 10 M_{\odot}\right)$ and are persistently luminous. A schematic sketch to scale of 21 of these systems is shown in Figure 1: The four at top are persistent systems and the 17 at bottom are the transients. For a review of the properties of black hole binaries, see Remillard \& McClintock (2006).

\footnotetext{
${ }^{1} 1 M_{\odot}=1$ solar mass $=2.0 \times 10^{33} \mathrm{~g}$.

${ }^{2} L_{\mathrm{Edd}}$ is the critical Eddington luminosity above which radiation pressure exceeds gravity.
} 


\section{The Continuum-Fitting Method}

A definite prediction of relativity theory is the existence of an innermost stable circular orbit (ISCO) for a particle orbiting a black hole. This inherently relativistic effect has a major impact on the structure of an accretion disk. At radii $R \geq R_{\mathrm{ISCO}}$ (the radius of the ISCO), accreting gas moves on nearly circular orbits and slowly spirals in toward the black hole. At the ISCO, however, the dynamics change suddenly and the gas, finding no more stable circular orbits, plunges into the hole. In the continuum-fitting (CF) method, one identifies the inner edge of the accretion disk with the ISCO (see Secs. 4 and 5 for supporting evidence) and estimates $R_{\mathrm{ISCO}}$ by fitting the X-ray continuum spectrum. Since the dimensionless radius $r_{\mathrm{ISCO}} \equiv R_{\mathrm{ISCO}} /\left(G M / c^{2}\right)$ is solely a monotonic function of the black hole spin parameter $a_{*}$ (Fig. 2$)^{3}$, knowing its value allows one immediately to infer the value of $a_{*}$. We note that the truncation of the disk at the ISCO is also a crucial assumption of the Fe K $\alpha$ method of measuring spin (Reynolds \& Fabian 2008).

Before describing the CF methodology, we stress that for this technique to succeed it is essential to have accurate measurements of the distance $D$ to the source, the inclination $i$ of the accretion disk, and the mass $M$ of the black hole (for reasons discussed below). The methodologies for measuring $D, i$ and $M$ are firmly established. Therefore, rather than digressing to discuss how these measurements are made, we refer the interested reader to some recent papers on the subject (Orosz et al. 2007, 2009, 2011; Cantrell et al. 2010).

The gaseous matter flowing from the companion star to the black hole has appreciable angular momentum as a consequence of the binary orbital motion. As the gas flows, viscous forces cause it to spread out into an orbiting structure known as an accretion disk. The gas flowing into the outer disk spirals slowly inward on Keplerian orbits on a time scale of weeks, reaching a typical temperature near the ISCO of $k T \sim 1 \mathrm{keV}$. Because the accretion disk is of fundamental importance to the measurement of black hole spin, we now describe in some detail the thin-disk model we employ.

The model we use is that described in Novikov \& Thorne (1973, hereafter NT), which is a relativisitic generalization of a Newtonian model developed by Shakura \& Sunvaev (1973). The NT model describes an axisymmetric radiatively-efficient accretion flow which, for a given black hole mass $M$, mass accretion rate $\dot{M}$ and black hole spin parameter $a_{*}$, gives a precise prediction for the local radiative flux $F(R)$ emitted at each radius $R$ of the disk. Moreover, the accreting gas is optically thick and the emission is thermal and blackbody-like,

\footnotetext{
${ }^{3}$ We express black hole spin in the customary way as the dimensionless quantity $a_{*} \equiv c J / G M^{2}$ with $\left|a_{*}\right| \leq 1$, where $M$ and $J$ are respectively the black hole mass and angular momentum.
} 
making it straightforward to compute the spectrum of the emission. Most importantly, the inner edge of the disk is located at the ISCO. Therefore, from the measurement of $R_{\mathrm{ISCO}}$, and if we know the mass $M$ of the black hole, we can immediately obtain $a_{*}$ (Fig. 2). This is the principle behind the CF method of estimating black hole spin, which was first described by Zhang et al. (1997).

Before discussing how to measure $R_{\mathrm{ISCO}}$ of a disk, we remind the reader how one measures the radius $R_{*}$ of a star. Given the distance $D$ to the star, the radiation flux $F_{\text {obs }}$ received from the star, and the temperature $T$ of its continuum radiation, the luminosity of the star is given by

$$
L_{*}=4 \pi D^{2} F_{\mathrm{obs}}=4 \pi R_{*}^{2} \sigma T^{4}
$$

where $\sigma$ is the Stefan-Boltzmann constant. From $F_{\text {obs }}$ and $T$, we immediately obtain the quantity $\pi\left(R_{*} / D\right)^{2}$, which is the solid angle subtended by the star. From this and the distance $D$, we immediately obtain $R_{*}$. For accurate results we must allow for limb darkening and other non-blackbody effects in the stellar emission by computing a stellar atmosphere model, but this is a detail.

The same principle applies to an accretion disk, but with some differences. First, since the flux $F(R)$ emitted locally by the disk varies with radius $R$, the radiation temperature $T(R)$ also varies with $R$. But the precise variation is known (if we assume the NT disk model), so it is easily incorporated into the model. Second, since the bulk of the emission is from the very inner regions of the disk, the effective area of the radiating surface is directly proportional to the square of the disk inner radius, $A_{\text {eff }}=C R_{\mathrm{ISCO}}^{2}$, where the constant $C$ is known. Third, the observed flux $F_{\text {obs }}$ depends not only on the luminosity and the distance, but also on the inclination $i$ of the disk to the line of sight. Allowing for these differences, one can write a relation for the disk problem similar in spirit to eq. (1), i.e., given $F_{\text {obs }}$ and a characteristic $T$ (from X-ray observations), one obtains the solid angle subtended by the ISCO: $\pi \cos i\left(R_{\mathrm{ISCO}} / D\right)^{2}$. If we further know $i$ and $D$, we obtain $R_{\mathrm{ISCO}}$; and if we also know $M$, we obtain $a_{*}$ (Fig. 2). This is the basic idea of the CF method.

There are three main issues that must be dealt with before applying the method: (1) One must carefully trace rays from the disk to the observer in the Kerr metric of the rotating black hole in order to compute accurately the observed flux and spectrum. To this end, we have developed an accretion disk model called KERRBB (Li et al. 2005) for fitting X-ray data. KERRBB assumes the NT model of the disk and carries out all the necessary ray-tracing to

\footnotetext{
${ }^{4}$ This model name and those that follow designate publicly-available programs that comprise a suite of X-ray data analysis software known as XSPEC (Arnaud 1996; http://heasarc.gsfc.nasa.gov/docs/xanadu/xspec/index.html).
} 
relate disk properties to observables. (2) One must have an accurate model for computing the spectral hardening factor $f=T / T_{\text {eff }}$, where $T$ is the temperature of the radiation at a given

radius and $T_{\text {eff }}$ is the effective temperature at the same radius defined by $F(R)=\sigma T_{\text {eff }}^{4}(R)$. This correction for non-blackbody effects is important at the high temperatures typically found in black hole disks. To carry out this correction we use the advanced disk atmosphere models of Davis et al. (2005) and Davis \& Hubeny (2006). (3) Most importantly, the inner accretion disk must be well described by the standard geometrically-thin, optically-thick NT disk model that we employ. To ensure this, we restrict our attention strictly to observations with a strong thermal component (Steiner et al. 2009a) and with disk luminosities below $30 \%$ of the Eddington limit (McClintock et al. 2006). In the two sections that follow, we present observational and theoretical evidence that at these luminosities the NT model is quite accurate.

For a full description of the mechanics of the CF method we refer the reader to Section 4 in McClintock et al. (2006). In brief, we fit the broadband X-ray continuum spectrum in conjunction with other components as needed, principally a Compton tail component that in more recent work is described using an empirical model of Comptonization called SIMPL (Steiner et al. 2009b). The accretion-disk component, which is key for the CF method, is modeled using KERRBB ( $\mathrm{Li}$ et al. 2005), which includes all relativistic effects within the context of the NT model, and also incorporates the advanced treatment of spectral hardening mentioned above. It furthermore includes self-irradiation of the disk ("returning radiation") and the effects of limb darkening. The key fit parameters returned are the black hole spin parameter $a_{*}$ and the mass accretion rate $\dot{M}$.

\section{Truncation of the Accretion Disk at the ISCO: Observational Evidence}

A crucial assumption that underlies both the $\mathrm{CF}$ and $\mathrm{Fe} \mathrm{K} \alpha$ approaches to measuring spin is that the accretion disk is quite sharply truncated at the ISCO. This assumption, which is fundamental to the NT model, is clearly valid if one considers only geodesic forces in the midplane. However, there are strong magnetohydrodynamic (MHD) forces acting in black hole accretion disks, and it is therefore unclear a priori that the disk terminates sharply at the ISCO. In this section, we present the observational evidence that there exists a fixed inner-disk radius in black hole binaries and, in the following section, we discuss the theoretical evidence for identifying this radius with $r_{\text {ISCO }}$.

There is a long history of evidence suggesting that fitting the X-ray continuum is a promising approach to measuring black hole spin. This history begins in the mid-1980s

with the application of a simple nonrelativistic multicolor disk model (Mitsuda et al. 1984; 
Makishima et al. 1986), now known as DISKBB, which returns the color temperature $T_{\text {in }}$ at the inner-disk radius $R_{\text {in }}$. In their review paper on black hole binaries, Tanaka \& Lewin (1995) summarize examples of the steady decay (by factors of 10-100) of the thermal flux of transient sources during which $R_{\text {in }}$ remains quite constant (see their Fig. 3.14). They remark that the constancy of $R_{\text {in }}$ suggests that this fit parameter is related to the radius of the ISCO. Zhang et al. (1997) then outlined how, using a relativistic disk model and corrections for the effects of radiative transfer, the fixed inner disk radius provides an observational basis to infer black hole spin. More recently, the evidence for a constant inner radius in the thermal state has been presented for a number of sources via plots showing that the bolometric luminosity of the thermal component is approximately proportional to $T_{\text {in }}^{4}$ (e.g., Kubota et al. 2001; Kubota \& Makishima 2004; Gierliński \& Done 2004; Abe et al. 2005; McClintock et al. 2009; Dunn et al. 2010).

A recent study of the persistent source LMC X-3 presents the most compelling evidence to date for a constant inner-disk radius (Steiner et al. 2010a). We analyzed many spectra collected during eight X-ray missions that span 26 years. As shown in Figure 3, for a selected sample of hundreds of spectra obtained using the Rossi X-ray Timing Explorer (RXTE), we find that to within $\approx 2$ percent the inner radius of the accretion disk is constant over time and unaffected by the gross variability of the source (top panel). Meanwhile, even considering an ensemble of eight X-ray missions, we find consistent values of the radius to within $\approx 4$ percent. These results provide compelling evidence for the existence of a fixed inner-disk radius and establish a firm foundation for the measurement of black hole spin. The only reasonable inference is that this radius is closely associated with the radius of the ISCO, as we show to be the case in the following section.

\section{Truncation of the Accretion Disk at the ISCO: Theoretical Evidence}

The relativistic NT model on which the CF method is currently built assumes, as does its predecessor the Newtonian model of Shakura \& Sunyaev (1973), that the accretion disk under consideration is geometrically thin. That is, the model assumes that the vertical thickness $H$ at any radius $R$ satisfies $H \ll R$. Assuming in addition that the disk is axisymmetric and in steady state, the model derives a number of relations which follow directly from basic conservation laws. One of the powerful results of this analysis is a formula for the disk flux profile $F(R)$ which depends only on the mass $M$ and spin $a_{*}$ of the black hole and the mass accretion rate $\dot{M}$, but is independent of messy details such as the viscosity of the accreting gas. It is the existence of this robust result for $F(R)$ that enables the CF method to work so well. 
There is, however, one unproven assumption in the NT model which is incorporated via a boundary condition: The model assumes that the shear stress (which drives the accretion at larger radii) vanishes at the ISCO. This "zero-torque" assumption is intuitively reasonable (since the gas switches to a rapidly plunging state once it crosses the ISCO, why should there be a stress at the transition radius?), but as NT themselves realized, it is ultimately an assumption. Paczyński (2000) and Afshordi \& Paczyński (2003) argued that deviations from the NT model decrease monotonically with decreasing disk thickness and that thin disks with $H / R \ll 1$ should be very well described by the model. Their argument, which was based on a hydrodynamical description of the disk, was confirmed by detailed calculations carried out by Shafee et al. (2008b). However, this leaves open the question of whether magnetized disks might deviate substantially from the NT model. In their paper, NT explicitly mention that magnetized disks could very well violate the zero-torque boundary condition.

Arguments have been advanced to suggest that a magnetized accreting gas will indeed have a non-zero shear stress at the ISCO (Krolik 1999; Gammie 1999), and that furthermore this stress could be so large that it may completely invalidate the NT model even in very thin disks. This is clearly an important question that strikes at the heart of the CF method. A number of recent studies of magnetized disks using three-dimensional general relativistic magnetohydrodynamic (GRMHD) simulations have explored this question (Shafee et al. 2008a; Revnolds \& Fabian 2008; Noble \& Krolik 2009; Noble et al. 2010; Penna et al. 2010). The conclusion of these authors is that the shear stress and the luminosity of the simulated disks do differ from the NT model, but perhaps not by a large amount.

Figure 4, taken from Kulkarni et al. (2010), shows the disk luminosity distribution $d L / d \ln R=4 \pi R^{2} F(R)$ derived from a set of four GRMHD thin-disk models simulated by Penna et al. (2010). These models have thicknesses $H / R \sim 0.045-0.08$ (see Penna et al. and Kulkarni et al. for a precise definition of $H$, which varies directly with luminosity). As is clear from the figure, the numerical models follow the NT model reasonably well, although they do deviate from it. Two kinds of deviation are seen. First, the numerical models produce some radiation inside the ISCO, whereas the NT model predicts no radiation there. Second, the peak of the emission in the simulated disks is shifted inward relative to the peak in the NT model. Both of these effects cause the disk to appear to have a smaller ISCO radius. This in turn means that, if one were to infer the black hole spin by fitting this luminosity distribution (or the corresponding spectrum) using the NT model, one would infer an erroneously large value for the spin. This systematic error arises because the NT model is not a perfect description of the simulated disk. To the extent that the simulated disk is a closer match to a real accretion disk than is the NT model, this allows us to estimate the corresponding systematic error in our measurements of spin. 
How serious is this systematic error? We answer this in three parts (see Kulkarni et al. 2010, for details).

1. Each of the models shown in Figure 4 causes an error in the spin estimate that is smallest for a low disk inclination (face-on disk; see Fig. 1) and largest for a high disk inclination $\left(i=75^{\circ}\right)$. For the latter (most unfavorable) case, the four models, which correspond to true spin values of $a_{*}=0,0.7,0.9$ and 0.98 , give via the CF method spin values of 0.17 , $0.83,0.936$ and 0.991 , respectively.

2. A similar exercise can be carried out for disks with other thicknesses. It is found that the error in the spin estimate is larger for thicker disks and smaller for thinner disks. Very roughly, the error appears to scale as $H / R$. Thus, the conclusion of Paczyński (2000), Afshordi \& Paczyński (2003) and Shafee et al. (2008b), that deviations from the NT model vanish in the limit of vanishingly small disk thickness, appears to be valid also for MHD disks.

3. The particular models shown in Figure 4 correspond to disk luminosities in the range $L / L_{\mathrm{Edd}} \sim 0.4-0.8$, based on their $H / R$. (It is difficult to be very quantitative since the mapping between luminosity and $H / R$ is not known precisely.) Since the CF method is applied only to observations at $L / L_{\text {Edd }}<0.3$, the systematic error due to inaccuracies in the theoretical model could be up to a factor of 2 smaller than the errors quoted in point 1 above.

Although the above results are based on numerical simulations that do not necessarily mimic real disks perfectly, we believe they still provide an estimate of the likely magnitude of the systematic error. The key point is that the level of systematic error we find is not serious at the current time. The observational errors considered in the following section are significantly larger in all cases. Stating this differently, while magnetized disks do behave as if their inner edges are shifted inward relative to the position of the ISCO (Fig. 4), the effect is quantitatively not serious for the disk luminosities (or disk thicknesses) at which the CF method is applied.

\section{Results of Continuum Fitting}

The spin results obtained to date for eight stellar-mass black holes are summarized in Table 1. The spins we find cover the full allowable range of prograde spins (Fig. 2) from $a_{*} \approx 0$ (Schwarzschild) to $a_{*} \approx 1$ (extreme Kerr). Interestingly, the spin values that have been obtained (Table 1), while spanning the full range of prograde spins, are all in the canonical physical range, namely $\left|a_{*}\right| \leq 1$. This is an important result and not at all a 
foregone conclusion: Given the hard external constraints on the dynamical model parameters ( $D, i$ and $M$; Section 3), it is entirely possible that a black hole will be found that implies a spin beyond the reach of our current model, i.e., $\left|a_{*}\right|>1$. Such a result could simply be caused by large systematic errors in $D, i$ and $M$. Or, more interestingly, it could falsify our model, or even possibly point to new physics. This is why we are excited about improving our measurements of $D, i$ and $M$ for the near-extreme Kerr hole GRS 1915+105 (see Sec. $6.2)$.

Error estimates are primitive for the first four spin results published in 2006 (Table 1). In recent work on the other four black holes, the principal sources of observational error, as well as the uncertainties in the key model parameters (e.g., the viscosity parameter), have been treated in detail. In particular, in our most recent paper on XTE J1550-564, we exhaustively explored many different sources of error (see Table 3 and Appendix A in Steiner et al. 2010b). The upshot of the work to date is that in every case the uncertainty in $a_{*}$ is completely dominated by the errors in the three key dynamical parameters that we input when fitting the X-ray spectral data. As discussed in Section 3, these parameters are the distance $D$, the black hole mass $M$, and the inclination of the inner disk $i$ (which we assume is aligned with the orbital angular momentum vector of the binary; Li et al. 2009). In order to determine the error in $a_{*}$ due to the combined uncertainties in $D, M$ and $i$, we perform Monte Carlo simulations assuming that these parameters are normally and independently distributed (e.g., see Gou et al. 2009). Note that the errors introduced by our use of the NT model (Sec. 5), which are not considered here, are significantly smaller than the observational errors (see Table 7 and Sec. 4 in Kulkarni et al. 2010).

We now discuss the results for four black holes in some detail. We first consider the persistent source M33 X-7. We then turn to GRS 1915+105, the prototype microquasar (Mirabel \& Rodríguez 1994), which hosts a near-extreme Kerr hole. Finally, we consider the microquasars A0620-00 and XTE J1550-564, contrasting their behavior with that of GRS $1915+105$.

\subsection{M33 X-7: The First Eclipsing Black Hole}

A long observation of the galaxy M33 with the Chandra X-ray Observatory led to the discovery of a black hole that is eclipsed by its companion star (Pietsch et al. 2006). We made a detailed follow-up dynamical study of the optical counterpart of M33 X-7, the first such study of a black hole binary beyond the environs of the Milky Way. We determined a precise mass for the black hole, $M=15.65 \pm 1.45 M_{\odot}$ (Orosz et al. 2007), as well as the mass of its exceptional companion star $\left(\approx 70 M_{\odot}\right)$. As we discuss in Orosz et al., it is difficult 
to understand the origin of this system - a massive black hole in a 3.5-day orbit, separated by only 42 solar radii from its supergiant companion (see Fig. 1). Recently, a consistent evolutionary model has been proposed that accounts for all the key properties of the system (Valsecchi et al. 2010). It assumes that M33 X-7 started as a primary of $\sim 95 M_{\odot}$ and a secondary of $\sim 30 M_{\odot}$, with an orbital period that is close to its present 3.5-day value.

Using as input our precise values for the black hole mass and orbital inclination angle, and the well-established distance of M33, we fitted 15 Chandra and XMM-Newton X-ray spectra and obtained a precise value for the spin of the black hole primary, $a_{*}=0.84 \pm 0.05$ (Liu et al. 2008, 2010). Remarkably, given that an (uncharged) astrophysical black hole is described by just its mass and spin, this result yields a complete description of an asteroidsize object at a distance of 2.74 million light-years ( $840 \mathrm{kp} 5$ ).

What is the origin of the spin of M33 X-7? Was the black hole born with its present spin, or was it torqued up gradually via the accretion flow supplied by its companion? In order to achieve a spin of $a_{*}=0.84$ via disk accretion, an initially non-spinning black hole must accrete $5.7 M_{\odot}$ from its donor star (King \& Kolb 1999) in becoming the $M=15.65 M_{\odot}$ black hole that we observe today. However, to transfer this much mass even in the case of Eddington-limited accretion requires $>17$ million years, 6 , whereas the massive companion star, and hence its host system, can not be older than about 2-3 million years (Orosz et al. 2007). Thus, it appears that the spin of M33 X-7 must be chiefly natal - i.e., the event horizon trapped much of the angular momentum of the collapsing stellar core - a conclusion that has been reached for two other stellar-mass black holes (McClintock et al. 2006; Shafee et al. 2006). (However, see Moreno Méndez et al. 2008 on hypercritical accretion).

\subsection{GRS 1915+105: A Near-Extreme Kerr Black Hole}

GRS 1915+105 has unique and striking properties that sharply distinguish it from the 50 or so known binaries that are believed to contain a stellar-mass black hole (McClintock et al. 2006; Özel et al. 2010). It is the most reliable source of relativistic radio jets in the Milky Way and is the prototype of the microquasars (Mirabel \& Rodríguez 1994). It frequently displays extraordinary X-ray variability that is not mimicked by any other black hole system. The properties of its high-frequency X-ray oscillations are equally extraordinary (Remillard \& McClintock 2006). Among the 17 transient black hole systems, GRS $1915+105$ is unique in having re-

\footnotetext{
${ }^{5} 1$ parsec $(\mathrm{pc})=3.26$ light-years.

${ }^{6} \dot{M}_{\mathrm{Edd}} \equiv L_{\mathrm{Edd}} / \eta c^{2}$, where $L_{\mathrm{Edd}}=1.3 \times 10^{39} \mathrm{M} / 10 M_{\odot} \mathrm{erg} \mathrm{s}^{-1}$ and the efficiency $\eta$ increases from $5.7 \%$ to $13.3 \%$ as $a_{*}$ increases from 0 to 0.84 (Shapiro \& Teukolsky 1983).
} 
mained active for more than a decade since its discovery in 1992. The system has an orbital period of 30.8 days, and it is the largest of the black hole binary systems (Fig. 1).

The pc-scale radio jets of GRS $1915+105$, with apparent velocities greater than the speed of light (superluminal motion), are the analogue of the kpc-scale jets that have long been observed for quasars. The source episodically ejects material at relativistic speeds, which can easily be tracked for weeks at centimeter wavelengths as clouds of plasma moving outward on the plane of the sky (Mirabel \& Rodríguez 1994; Fender et al. 1999). Based on a kinematic model, the jet velocity for a plausible distance of $D \sim 11 \mathrm{kpc}$ is $v_{\mathrm{J}} / c>0.9$, and the inclination of the jet from our line of sight is $i_{\mathrm{J}} \approx 65^{\circ}$.

Based on the analysis of X-ray spectral data for reasonable estimates of $D, M$ and $i$, we discovered that GRS $1915+105$ contains a near-extreme Kerr hole with $a_{*}>0.98$ (McClintock et al. 2006). However, the current estimates of both $D$ and $M$ are poor: The distance is uncertain by a factor of $\approx 2$ (Fig. $5 b$ ), and the mass is uncertain by $\approx 30 \%$ $\left(M=14.4 \pm 4.4 M_{\odot}\right.$; Greiner et al. 2001; Harlaftis \& Greiner 2004).

Remarkably, the extraordinarily high spin of GRS 1915+105 and other properties of the source allow one - for this source only - to place tight constraints on the allowable values of $M$ and $D$, which must lie within the triangular region shown in Figure $5 a$. Our spin model constrains the black hole's mass and distance to lie to the right of the slanted line (99\% confidence level) because the model implies values of $a_{*}>1$ to the left of the line. (Our model is only valid for $a_{*}<0.999$ ). Distances $>12 \mathrm{kpc}$ are ruled out by the kinematic model of the radio jets (Fender et al. 1999). The lower bound on $M$ is an estimate and is based on work in progress: We (Danny Steeghs et al.) are in the act of obtaining nearinfrared spectroscopic data at ESO's VLT Observatory that we fully expect will improve the measurement of $M$ by at least a factor of two. When this result is in hand, we will have $M$ and $D$ constrained to lie within the small shaded triangle, thereby constraining the distance to lie within the range $9.5-12 \mathrm{kpc}$.

As noted above, the distance is also highly uncertain. Furthermore, all measurements to date are model-dependent estimates with large, systematic uncertainties that are difficult to assess (Fig. 5b). With Mark Reid, we are in the act of obtaining a model-independent trigonometric distance with an uncertainty of $10 \%$ via a parallax measurement (the goldstandard method in astronomy) using the Very Long Baseline Array (VLBA), a worldwide array of radio telescopes. We have made successful observations at four epochs in 2008-2010 and anticipate that observations at several additional epochs will be required to reach our goal. Two hypothetical and possible outcomes of these VLBA observations are indicated in Figure 5a: VLBA2 would confirm our spin model and VLBA1 would rule it out. 


\subsection{A0620-00 and XTE J1550-564: Two Schwarzschild-Like Black Holes}

The host systems of these two black holes are quite small (Fig. 1). The optical companion in A0620-00, which has an orbital period of only 0.3 days, is a star somewhat cooler than the Sun with about half its size and mass. During its yearlong X-ray outburst in 1975-1976, this nearby transient $(D \approx 1 \mathrm{kpc}$ ) became the brightest celestial X-ray source ever observed (apart from the Sun). For several days, the flux at Earth from this source was greater than the combined flux of all of the hundreds of other X-ray binaries in our galaxy. During this period, A0620-00 was also a bright transient radio source, which was observed with the early radio telescopes of the day. A reanalysis of these data by Kuulkers et al. (1999) indicates that multiple jet ejections occurred. The authors find that, like GRS 1915+105, the radio source was extended on parsec scales, and they infer a relativistic expansion velocity.

The cool companion star in XTE J1550-564 also has a mass about half that of the Sun, although its radius is about twice as great (Fig. 1); the orbital period of the system is 1.5 days (Orosz et al. 2011). During its principal 1998-1999 outburst cycle, this transient source produced one of the most remarkable X-ray flare events ever observed for a black hole binary. For $\approx 1$ day, the source intensity rose fourfold and the flux in the dominant nonthermal component of emission rose by the same factor. Then, just as quickly, the source intensity declined to its pre-outburst level (Sobczak et al.|2000). Four days later, small-scale superluminal radio jets were observed (Hannikainen et al. 2009); their separation angle and relative velocity link them to the impulsive X-ray flare. The subsequent detection of pc-scale radio jets in 2000 led to the discovery of relativistic $X$-ray jets (Corbel et al. 2002). All of the available evidence strongly indicates that these pc-scale X-ray and radio jets are associated with the powerful X-ray flare.

Using our recently-determined estimates of $D, M$ and $i$ for A0620-00 and XTE J1550564 (Cantrell et al. 2010; Orosz et al. 2011), we fitted the X-ray spectra of these black holes and determined their spins (Table 1). Figure 6 shows a pair of fitted spectra for the latter source. The spectrum in the left panel is completely dominated by the thermal component and is therefore ideal for the determination of spin. Now, however, using our improved methodologies (Steiner et al. 2009a, 2010b), we are able to obtain useful and consistent values of spin as well for spectra that have a strong Compton component of emission, like the one shown in the right panel of Figure 6. The origin of this component is widely attributed to Compton upscattering of the soft disk photons by coronal electrons (see Sec. 7).

The CF spins of both A0620-00 and XTE J1550-564 are quite low: $a_{*} \approx 0.1$ and $a_{*} \approx 0.3$, respectively. The corresponding nominal radii of their ISCOs are $5.7 M$ and $5.0 M$, which differ only modestly from the Schwarzschild value of $6 M$. The low spins of these two microquasars challenge the long-standing and widely-held belief that there is a strong 
connection between black hole spin and relativistic jets (Blandford \& Znajek 1977, hereafter BZ). If relativistic jets are powered by black hole spin, then theory predicts that jet power

will increase dramatically with increasing $a_{*}$ (Tchekhovskoy et al. 2010). For low spins, the black hole contributes very little power; in fact, for $a_{*}<0.4$, the accretion disk apparently provides more power than the black hole (McKinney 2005).

Given the low spins of XTE J1550-564 and A0620-00, it would appear that their episodic jets are driven largely by their accretion disks. One well-known candidate mechanism is the centrifugally driven outflow of matter from a disk described by Blandford \& Payne (1982, hereafter BP). A useful comparison of the operational regimes of BP and BZ is given by Garofalo et al. (2010). They show that BP is always viable, but that BZ is a more likely mechanism for the most rapidly rotating sources, such as the extreme-Kerr black hole GRS 1915+105 (see Sec. 6.2). In closing, we note that a statistical study by Fender et al. (2010), which is based on data of uneven quality, found no evidence that black hole spin powers jets.

\section{The Fe $\mathbf{K} \alpha$ Reflection Method}

In the Fe K $\alpha$ method, one determines $r_{\text {ISCO }}$ by modeling the profile of the broad and skewed iron line, which is formed in the inner disk by Doppler effects, light bending, relativistic beaming, and gravitational redshift (Fabian et al. 2000; Reynolds \& Nowak 2003; Miller 2007). Of central importance is the effect of the gravitational redshift on the red wing of the line. This wing extends to very low energies for a rapidly rotating black hole $\left(a_{*} \sim 1\right)$ because in this case gas can orbit near the event horizon, deep in the potential well of the black hole. Relative to the CF method, measuring the extent of the red wing in order to infer $a_{*}$ is hindered by the relative faintness of the signal. However, the Fe K $\alpha$ method has the virtues that it is independent of $M$ and $D$, while the blue wing of the line even allows an estimate if $i$. As noted in Section 1, this method, while applicable to both classes of black holes, is presently the only viable approach to measuring the spins of supermassive black holes.

For stellar-mass black holes, in addition to the thermal disk component of emission (which is central to the CF method), a higher-energy power-law component of emission is always observed (e.g., see Fig. 6). For supermassive black holes in active galactic nuclei (AGN), this power-law component is dominant, and it is thought to be produced by inverse Compton scattering of soft thermal photons in a hot $(k T \sim 100 \mathrm{keV})$ corona (Reynolds \& Nowak 2003; Done et al. 2007). Meanwhile, the disks of both stellar-mass and supermassive black holes are too cool $(k T \sim 1$ and $0.01 \mathrm{keV}$, respectively) to produce the observed Fe $\mathrm{K} \alpha$ 
emission line. Rather, this dominant line, and a host of other lines, are generated via X-ray fluorescence as a result of irradiation of the disk by the hard, coronal power-law component. The complex spectrum so generated is referred to as a "reflection spectrum." In order to determine the spin using the Fe-line method, one must model the reflection spectrum in detail (Ross \& Fabian 2005, 2007).

The spins of several stellar-mass black holes have been measured using the $\mathrm{Fe} \mathrm{K} \alpha$ method. An early suggestion of high spins for two black holes was made by Miller et al. (2002, 2004) and preliminary results for a total of eight stellar-mass black holes are given in Miller et al. (2009). Other important papers on the spins of stellar-mass black holes include Reis et al. (2008, 2009). For a review, see Miller (2007). Very recently, we teamed up with Fe K $\alpha$ experts to measure the spin of XTE J1550-564 (Steiner et al. 2010b). The spin estimate obtained using the Fe K $\alpha$ method is $a_{*}=0.55_{-0.22}^{+0.15}$, which is quite consistent with the CF value (see Table 1 ).

The spins of several supermassive black holes have been reported, which range from $a_{*} \approx 0.6$ to $>0.98$ (Brenneman \& Revnolds 2006; Fabian et al. 2009; Schmoll et al. 2009; Miniutti et al. 2009). By far, the most well studied of these is the Seyfert 1 galaxy MCG-630-15 (for background, see Reynolds \& Nowak 2003). The $6.4 \mathrm{keV}$ Fe K $\alpha$ line of this AGN is extremely broad and skewed. Brenneman \& Reynolds (2006) and Miniutti et al. (2009) show that the red wing extends downward to below $4 \mathrm{keV}$ and conclude that $a_{*}>0.98$.

\section{Conclusion}

We have discussed the only two established classes of black holes, stellar-mass and supermassive 7 , and the two principal approaches to measuring their spins, the continuum-fitting and Fe-K $\alpha$ methods. Spin measurements for eight stellar-mass black holes are presented, and these data are used to argue that the high spin of M33 X-7 is natal, and that at least some relativistic black-hole jets are powered by their accretion disks, not the spin of the black hole.

Two aspects of this work excite us greatly.

First, by measuring a black hole's spin, after earlier measuring its mass, we are able to completely characterize the intrinsic properties of each of the black holes we study. The No

\footnotetext{
${ }^{7}$ There is evidence for a class of intermediate-mass $\left(100-10^{5}\right)$ black holes (Miller \& Colbert 2004). However, to date there existence remains uncertain because no direct and confirming measurement of mass has been made.
} 
Hair Theorem states that a macroscopic black hole, regardless of how massive it may be, is described by just two parameters: $M$ and $a_{*} 8$. But is the No Hair Theorem really true? The only way we will answer this question is by first measuring $M$ and $a_{*}$ for a good sample of black holes, and then testing whether the Kerr metric corresponding to these values of $M$ and $a_{*}$ is completely consistent with all observables that are sensitive to the space-time near the black hole. In a sense, we have attempted the first test of the No Hair Theorem by measuring the spin of XTE J1550-564 by two independent methods, the continuum fitting method and the Fe K $\alpha$ method (Sec. 7), and finding agreement. However, the errors in the two measurements are currently rather large, and we do not yet understand all systematic sources of error, so it would be premature to claim success. But this example provides a taste of how astrophysics can contribute to deep questions in physics.

The other aspect that excites us is all the areas of astrophysics that our work ties to, e.g., the connections that are beginning to be made between measurements of spin and the phenomenology and theory of relativistic jets (Sec. 6.3), and the processes that lead to black hole formation (Sec. 6.1). We hope to see spin data used to help constrain models of gammaray bursts, black hole formation, black-hole binary evolution, high- and low-frequency X-ray oscillations, black hole X-ray states and state transitions, models of X-ray coronae, etc.

These two strong motivations stimulate us to continue firming up the measurements of black hole spin, with the goal of amassing a good sample of a total of 12-15 measurements during the next several years.

We conclude by noting that it is reasonable to expect LIGO, LISA and other gravitationalwave observatories to provide us with intimate knowledge concerning black holes. However, these breakthroughs are still some years in the future whereas astrophysical techniques are providing information on black holes today. Also, gravitational-wave facilities are unlikely to help us understand MHD accretion flows in strong fields, or the origin of relativistic jets, or the formation of relativistically-broadened Fe lines and high-frequency quasi-periodic oscillations, etc., phenomena that are now routinely observed for black holes. In short, observations of accreting black holes show us uniquely how a black hole interacts with its environment.

There is no straight path to unlocking the mysteries of black holes, probing the extreme physical conditions they generate, and understanding their importance to astrophysics and cosmology. It behooves us to explore widely because it is the synergistic exploration of all paths that will enlighten us. Therefore, it is important to maintain balance between gravitational-wave and electromagnetic studies of black holes.

\footnotetext{
${ }^{8}$ In principle it could also have an electric charge, but astrophysical black holes are unlikely to have enough charge to be dynamically important.
} 
J.E.M. acknowledges support from NASA grant NNX08AJ55G and the Smithsonian Endowment Funds. R.N. was supported in part by NSF grant AST-0805832 and NASA grant NNX08AH32G. We thank Laura Brenneman and Jon Miller for helpful comments on the Fe-line method. 
Table 1. Spin Results to Date for Eight Black Holes ${ }^{\mathrm{a}}$

\begin{tabular}{llll}
\hline \hline & \multicolumn{1}{c}{ Source } & \multicolumn{1}{c}{ Spin $a_{*}$} & \multicolumn{1}{c}{ Reference } \\
\hline 1 & GRS 1915+105 & $>0.98$ & McClintock et al. 2006 \\
2 & LMC X-1 & $0.92_{-0.07}^{+0.05}$ & Gou et al. 2009 \\
4 & M33 X-7 & $0.84 \pm 0.05$ & $\underline{\text { Liu et al. 2008, 2010 }}$ \\
3 & 4 U 1543-47 & $0.80 \pm 0.05$ & $\underline{\underline{\text { Shafee et al. 2006 }}}$ \\
5 & GRO J1655-40 & $0.70 \pm 0.05$ & $\underline{\underline{\text { Shafee et al. 2006 }}}$ \\
6 & XTE J1550-564 & $0.34_{-0.28}^{+0.20}$ & $\underline{\underline{\text { Steiner et al. 2010b }}}$ \\
7 & LMC X-3 & $<0.3^{\mathrm{b}}$ & Davis et al. 2006 \\
8 & A0620-00 & $0.12 \pm 0.18$ & $\underline{\text { Gou et al. 2010 }}$ \\
\hline
\end{tabular}

${ }^{a}$ Errors are quoted at the $68 \%$ level of confidence.

${ }^{\mathrm{b}}$ Provisional result pending improved measurements of $M$ and $i$. 


\section{REFERENCES}

Abe, Y., Fukazawa, Y., Kubota, A., Kasama, D., \& Makishima, K. 2005, PASJ, 57, 629

Afshordi, N. \& Paczyński, B. 2003, ApJ, 592, 354

Arnaud, K. A. 1996, in Astronomical Society of the Pacific Conference Series, Vol. 101, Astronomical Data Analysis Software and Systems V, ed. G. H. Jacoby \& J. Barnes, 17

Barai, P., Das, T. K., \& Wiita, P. J. 2004, ApJ, 613, L49

Blandford, R. D. \& Payne, D. G. 1982, MNRAS, 199, 883

Blandford, R. D. \& Znajek, R. L. 1977, MNRAS, 179, 433

Brenneman, L. W. \& Reynolds, C. S. 2006, ApJ, 652, 1028

Cantrell, A. G. et al. 2010, ApJ, 710, 1127

Corbel, S., Fender, R. P., Tzioumis, A. K., Tomsick, J. A., Orosz, J. A., Miller, J. M., Wijnands, R., \& Kaaret, P. 2002, Science, 298, 196

Davis, S. W., Blaes, O. M., Hubeny, I., \& Turner, N. J. 2005, ApJ, 621, 372

Davis, S. W., Done, C., \& Blaes, O. M. 2006, ApJ, 647, 525

Davis, S. W. \& Hubeny, I. 2006, ApJS, 164, 530

Done, C., Gierliński, M., \& Kubota, A. 2007, A\&A Rev., 15, 1

Dovčiak, M., Muleri, F., Goosmann, R. W., Karas, V., \& Matt, G. 2008, MNRAS, 391, 32

Dunn, R. J. H., Fender, R. P., Körding, E. G., Belloni, T., \& Cabanac, C. 2010, MNRAS, 403, 61

Fabian, A. C., Iwasawa, K., Reynolds, C. S., \& Young, A. J. 2000, PASP, 112, 1145

Fabian, A. C. et al. 2009, Nature, 459, 540

Fender, R. P., Gallo, E., \& Russell, D. 2010, MNRAS, 406, 1425

Fender, R. P., Garrington, S. T., McKay, D. J., Muxlow, T. W. B., Pooley, G. G., Spencer, R. E., Stirling, A. M., \& Waltman, E. B. 1999, MNRAS, 304, 865

Gammie, C. F. 1999, ApJ, 522, L57 
Garofalo, D., Evans, D. A., \& Sambruna, R. M. 2010, MNRAS, 406, 975

Gierliński, M. \& Done, C. 2004, MNRAS, 347, 885

Gou, L. et al. 2009, ApJ, 701, 1076

Gou, L., McClintock, J. E., Steiner, J. F., Narayan, R., Cantrell, A. G., Bailyn, C. D., \& Orosz, J. A. 2010, ApJ, 718, L122

Greiner, J., Cuby, J. G., \& McCaughrean, M. J. 2001, Nature, 414, 522

Hannikainen, D. C. et al. 2009, MNRAS, 397, 569

Harlaftis, E. T. \& Greiner, J. 2004, A\&A, 414, L13

Huang, L., Cai, M., Shen, Z., \& Yuan, F. 2007, MNRAS, 379, 833

King, A. R. \& Kolb, U. 1999, MNRAS, 305, 654

Krolik, J. H. 1999, ApJ, 515, L73

Kubota, A. \& Makishima, K. 2004, ApJ, 601, 428

Kubota, A., Makishima, K., \& Ebisawa, K. 2001, ApJ, 560, L147

Kulkarni, A. K. et al. 2010, MNRAS submitted

Kuulkers, E., Fender, R. P., Spencer, R. E., Davis, R. J., \& Morison, I. 1999, MNRAS, 306, 919

Li, L., Narayan, R., \& McClintock, J. E. 2009, ApJ, 691, 847

Li, L., Zimmerman, E. R., Narayan, R., \& McClintock, J. E. 2005, ApJS, 157, 335

Liu, J., McClintock, J. E., Narayan, R., Davis, S. W., \& Orosz, J. A. 2008, ApJ, 679, L37

- 2010, ApJ, 719, L109

Makishima, K., Maejima, Y., Mitsuda, K., Bradt, H. V., Remillard, R. A., Tuohy, I. R., Hoshi, R., \& Nakagawa, M. 1986, ApJ, 308, 635

McClintock, J. E., Remillard, R. A., Rupen, M. P., Torres, M. A. P., Steeghs, D., Levine, A. M., \& Orosz, J. A. 2009, ApJ, 698, 1398

McClintock, J. E., Shafee, R., Narayan, R., Remillard, R. A., Davis, S. W., \& Li, L. 2006, ApJ, 652, 518 
McKinney, J. C. 2005, ApJ, 630, L5

Miller, J. M. 2007, ARA\&A, 45, 441

Miller, J. M. et al. 2004, ApJ, 606, L131

- 2002, ApJ, 570, L69

Miller, J. M., Reynolds, C. S., Fabian, A. C., Miniutti, G., \& Gallo, L. C. 2009, ApJ, 697, 900

Miller, M. C. \& Colbert, E. J. M. 2004, International Journal of Modern Physics D, 13, 1

Miniutti, G., Panessa, F., de Rosa, A., Fabian, A. C., Malizia, A., Molina, M., Miller, J. M., \& Vaughan, S. 2009, MNRAS, 398, 255

Mirabel, I. F. \& Rodríguez, L. F. 1994, Nature, 371, 46

Mitsuda, K. et al. 1984, PASJ, 36, 741

Moreno Méndez, E., Brown, G. E., Lee, C., \& Park, I. H. 2008, ApJ, 689, L9

Noble, S. C. \& Krolik, J. H. 2009, ApJ, 703, 964

Noble, S. C., Krolik, J. H., \& Hawley, J. F. 2010, ApJ, 711, 959

Novikov, I. D. \& Thorne, K. S. 1973, in Black Holes (Les Astres Occlus), ed. C. Dewitt \& B. S. Dewitt, (New York: Gordon \& Breach), 343-450

Orosz, J. A. et al. 2007, Nature, 449, 872

- 2009, ApJ, 697, 573

Orosz, J. A., Steiner, J., McClintock, J. E., Torres, M. A. P., Remillard, R. A., \& Bailyn, C. D. 2011, ApJ in press, arXiv:1101.2499v1 [astro-ph.SR]

Özel, F., Psaltis, D., Narayan, R., \& McClintock, J. E. 2010, ApJ, 725, 1918

Paczyński, B. 2000, arXiv:astro-ph/0004129v1

Penna, R. F., McKinney, J. C., Narayan, R., Tchekhovskoy, A., Shafee, R., \& McClintock, J. E. 2010, MNRAS, 408, 752

Pietsch, W., Haberl, F., Sasaki, M., Gaetz, T. J., Plucinsky, P. P., Ghavamian, P., Long, K. S., \& Pannuti, T. G. 2006, ApJ, 646, 420 
Reis, R. C., Fabian, A. C., Ross, R. R., \& Miller, J. M. 2009, MNRAS, 395, 1257

Reis, R. C., Fabian, A. C., Ross, R. R., Miniutti, G., Miller, J. M., \& Reynolds, C. 2008, MNRAS, 387, 1489

Remillard, R. A. \& McClintock, J. E. 2006, ARA\&A, 44, 49

Reynolds, C. S. \& Fabian, A. C. 2008, ApJ, 675, 1048

Reynolds, C. S. \& Nowak, M. A. 2003, Phys. Rep., 377, 389

Ross, R. R. \& Fabian, A. C. 2005, MNRAS, 358, 211

- 2007, MNRAS, 381, 1697

Schmoll, S. et al. 2009, ApJ, 703, 2171

Shafee, R., McClintock, J. E., Narayan, R., Davis, S. W., Li, L., \& Remillard, R. A. 2006, ApJ, 636, L113

Shafee, R., McKinney, J. C., Narayan, R., Tchekhovskoy, A., Gammie, C. F., \& McClintock, J. E. 2008a, ApJ, 687, L25

Shafee, R., Narayan, R., \& McClintock, J. E. 2008b, ApJ, 676, 549

Shakura, N. I. \& Sunyaev, R. A. 1973, A\&A, 24, 337

Shapiro, S. L. \& Teukolsky, S. A. 1983, Black holes, white dwarfs, and neutron stars: The physics of compact objects, ed. Shapiro, S. L. \& Teukolsky, S. A. (New York: Wiley)

Shcherbakov, R. V. \& Huang, L. 2011, MNRAS, 410, 1052

Sobczak, G. J., McClintock, J. E., Remillard, R. A., Cui, W., Levine, A. M., Morgan, E. H., Orosz, J. A., \& Bailyn, C. D. 2000, ApJ, 544, 993

Steiner, J. F., McClintock, J. E., Remillard, R. A., Gou, L., Yamada, S., \& Narayan, R. 2010a, ApJ, 718, L117

Steiner, J. F., McClintock, J. E., Remillard, R. A., Narayan, R., \& Gou, L. 2009a, ApJ, 701, L83

Steiner, J. F., Narayan, R., McClintock, J. E., \& Ebisawa, K. 2009b, PASP, 121, 1279

Steiner, J. F. et al. 2010b, MNRAS submitted, arXiv:1010.1013v2 [astro-ph.HE] 
Suleimanov, V. F., Lipunova, G. V., \& Shakura, N. I. 2008, A\&A, 491, 267

Swank, J., Kallman, T., \& Jahoda, K. 2008, in COSPAR, Plenary Meeting, Vol. 37, 37th COSPAR Scientific Assembly, 3102

Takahashi, R. 2004, ApJ, 611, 996

Tanaka, Y. \& Lewin, W. H. G. 1995, in X-ray Binaries, ed. W. H. G. Lewin, J. van Paradijs, \& E. P. J. van den Heuvel, (Cambridge: Cambridge Univ. Press), 126-174

Tchekhovskoy, A., Narayan, R., \& McKinney, J. C. 2010, ApJ, 711, 50

Török, G., Abramowicz, M. A., Kluźniak, W., \& Stuchlík, Z. 2005, A\&A, 436, 1

Valsecchi, F., Glebbeek, E., Farr, W. M., Fragos, T., Willems, B., Orosz, J. A., Liu, J., \& Kalogera, V. 2010, Nature, 468, 77

Wagoner, R. V., Silbergleit, A. S., \& Ortega-Rodríguez, M. 2001, ApJ, 559, L25

Zhang, S. N., Cui, W., \& Chen, W. 1997, ApJ, 482, L155 


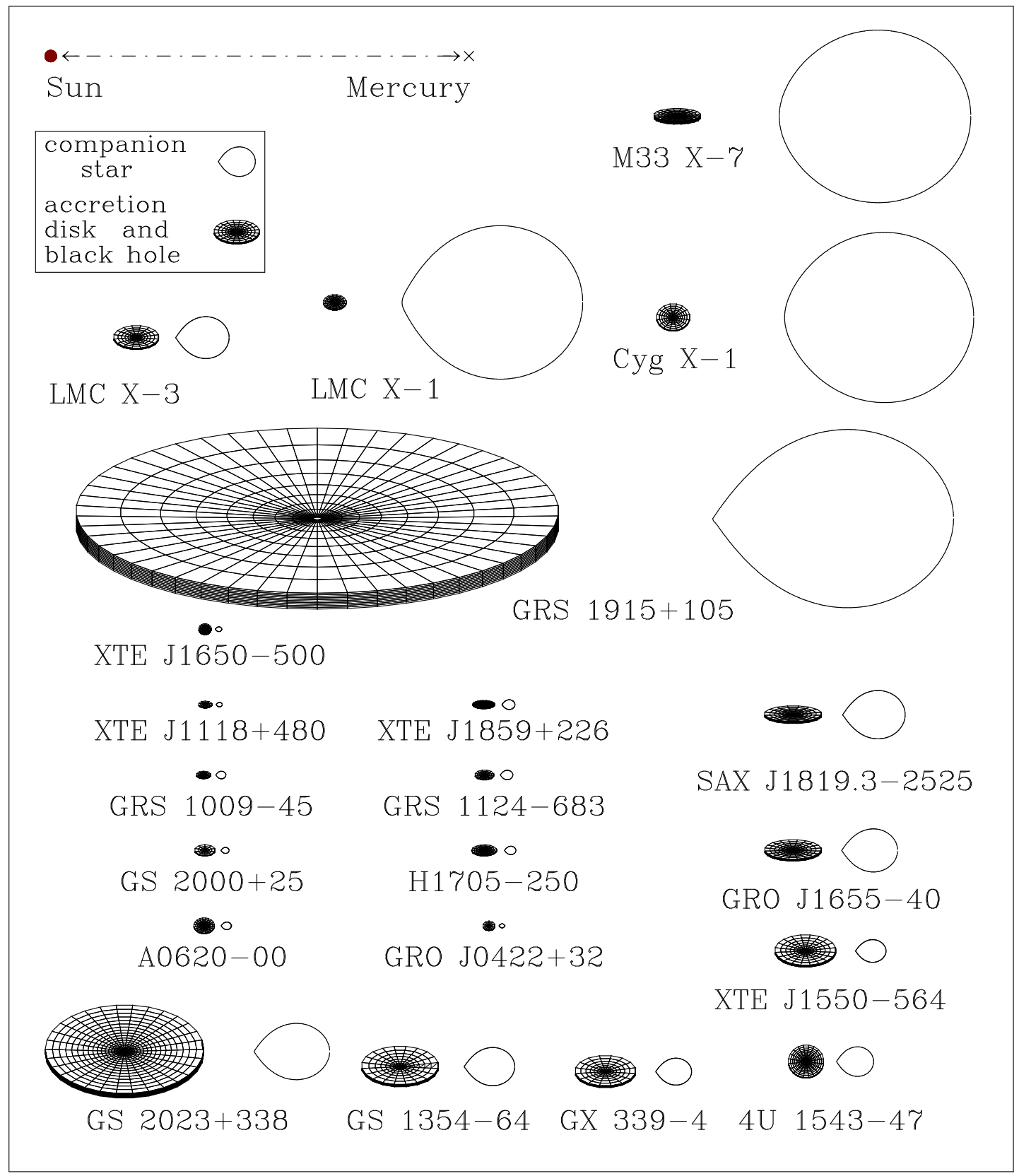

Fig. 1.- Scale drawings of 21 black hole binaries. The size of the Sun and the SunMercury distance $(0.4 \mathrm{AU})$ are indicated at the top. The systems range in size from the giant GRS $1915+105$ with an orbital period of 30.8 days to tiny XTE J1118+480 with an orbital period of 0.2 days. The shapes of the tidally distorted stars are accurately rendered, and the black hole is located at the center of the accretion disk (see key in inset). The inclination of the binary to our line of sight is indicated by the tilt of the accretion disk; an inclination angle of $i=0^{\circ}$ corresponds to a system whose accretion disk lies in the plane of the sky and is viewed face on (e.g., $i=21^{\circ}$ for $4 \mathrm{U} 1543-47$ and $i=75^{\circ}$ for SAX J1819.3-2525). 


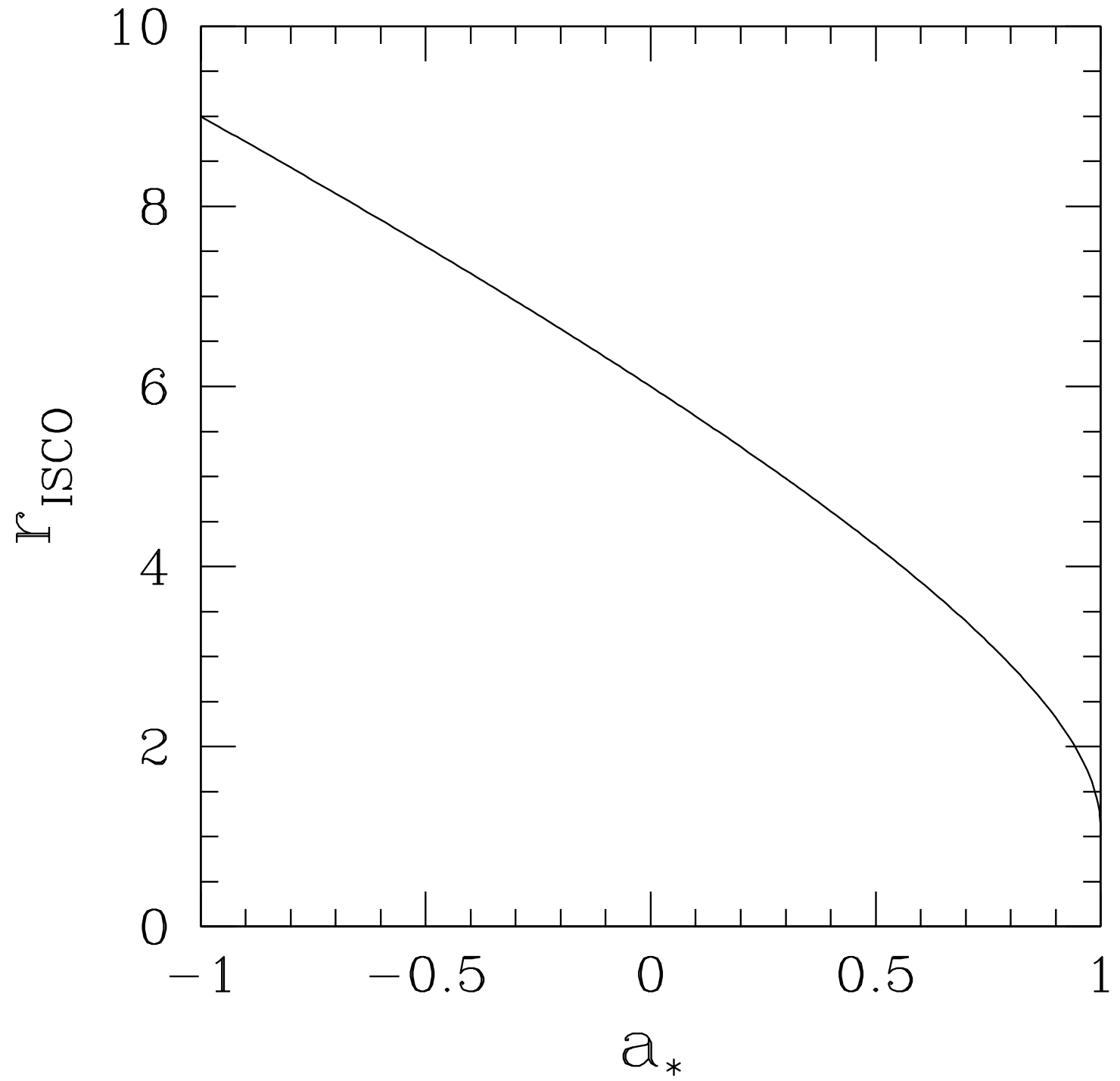

Fig. 2.- Radius of the ISCO in units of $G M / c^{2}$ versus the black hole spin parameter. Negative values of $a_{*}$ correspond to retrograde motion, with the black hole spinning in the opposite sense of the disk. Stellar black holes are expected to have prograde spins $\left(a_{*}>0\right)$ as a consequence of their formation in a binary system, whereas the spins of supermassive black holes, which are conditioned by galaxy merger events, may be either prograde or retrograde (e.g., Garofalo et al. 2010). 


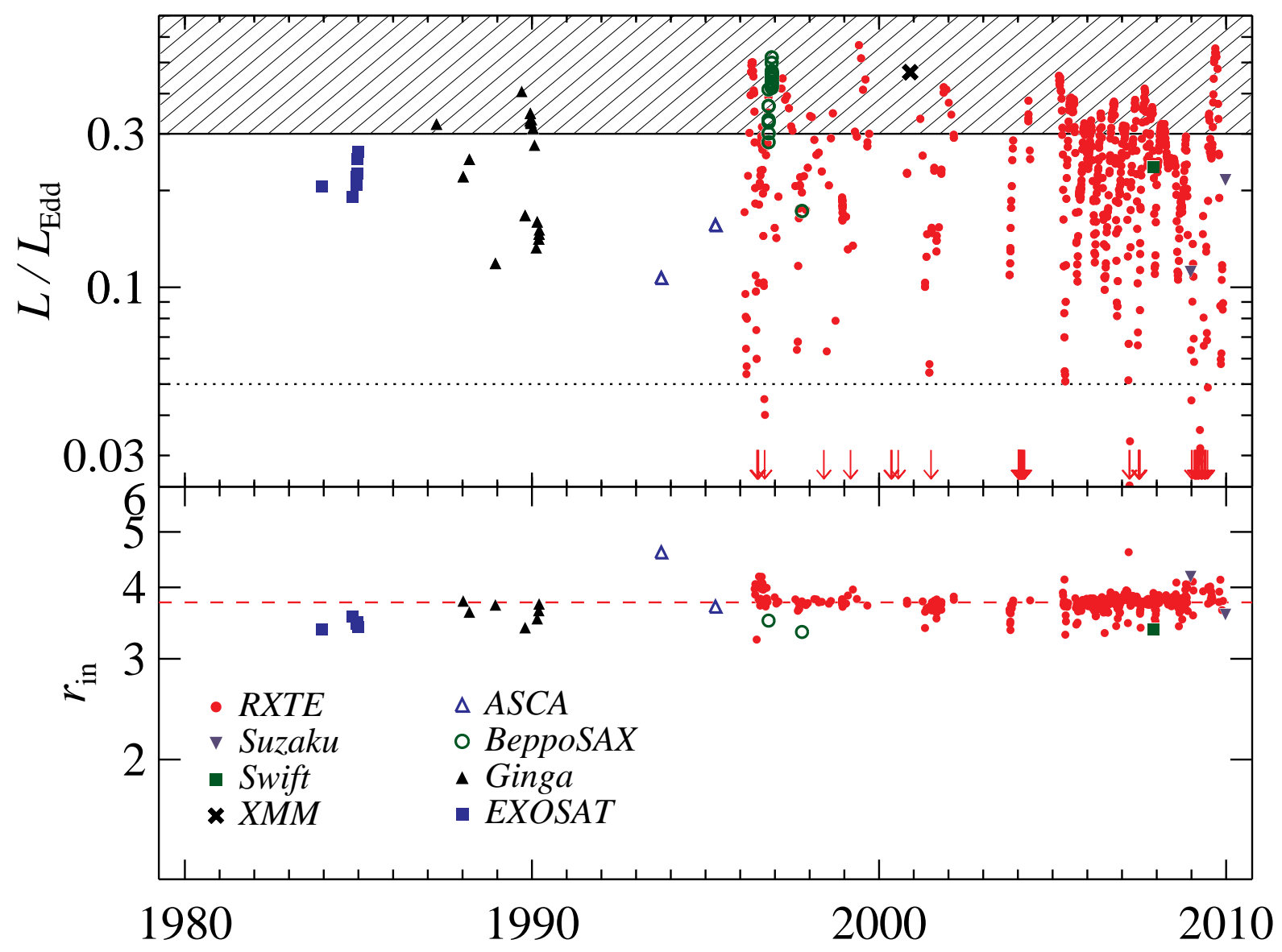

Fig. 3.- (top) Accretion-disk luminosity in Eddington-scaled units (for $M=10 M_{\odot}$ ) versus time for all the 766 spectra considered in the study of LMC X-3 by Steiner et al. (2010a). The downward arrows show RXTE data which are off scale. Data in the unshaded region satisfy our thin-disk selection criterion $L / L_{\mathrm{Edd}}<0.3$ (Sec. 3). The dotted line indicates the lower luminosity threshold $\left(5 \% \mathrm{~L} / L_{\mathrm{Edd}}\right)$ set to avoid confusion with strongly Comptonized data. (bottom) Fitted values of the inner disk radius $r_{\text {in }} \equiv R_{\text {in }} /\left(G M / c^{2}\right)$ are shown for thin-disk data in the top panel that meet the selection criteria of the study (a total of 411 spectra). Despite large variations in luminosity, $r_{\text {in }}$ remains constant to within a few percent over time. The median value for just the 391 selected $R X T E$ spectra is shown as a red dashed line. 


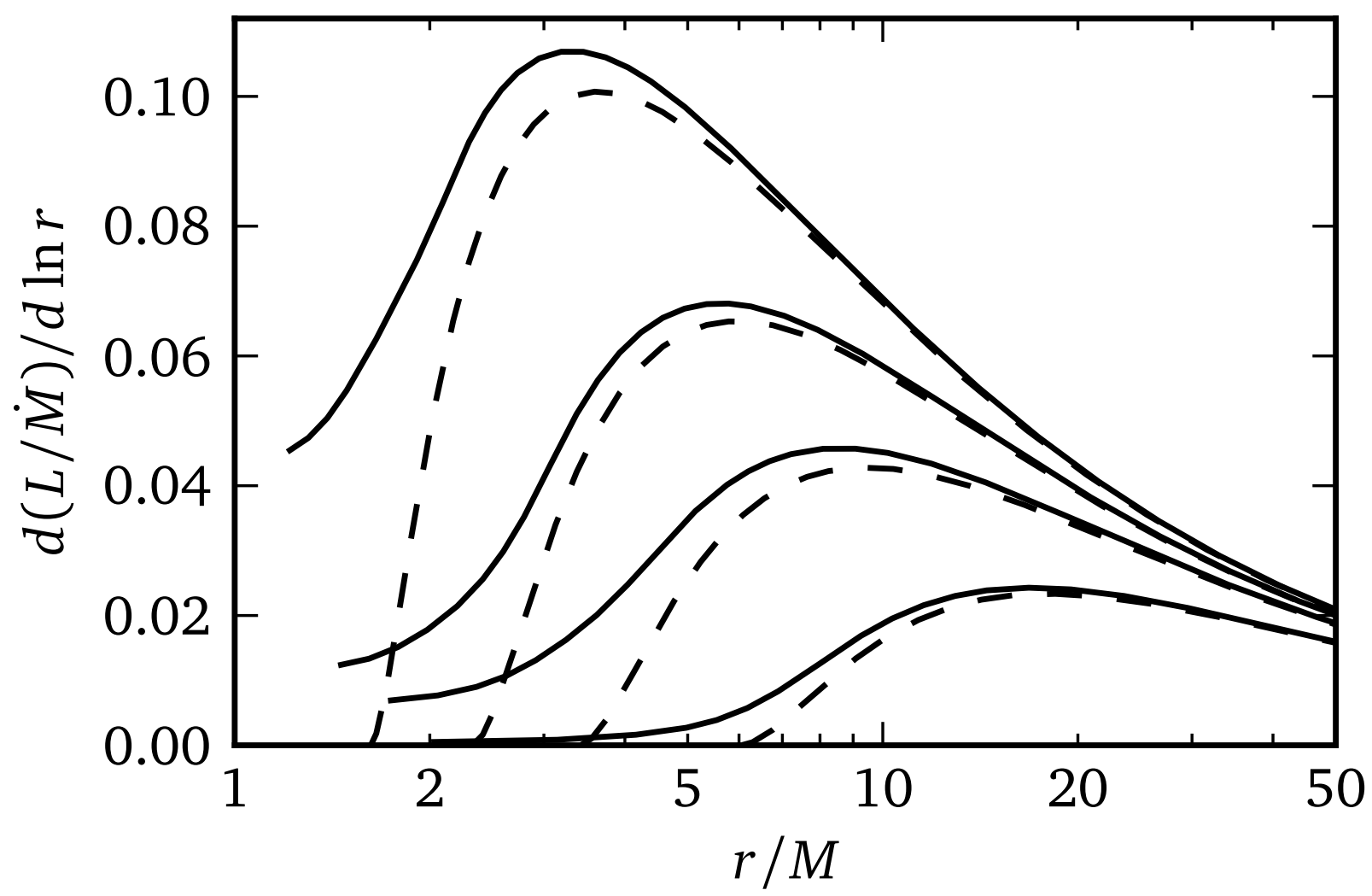

Fig. 4.- Luminosity profiles from GRMHD simulations (solid lines) compared with those from the Novikov \& Thorne model (dashed lines) for $a_{*}=0,0.7,0.9$ and 0.98 (bottom to top). The ISCO is located at the radius where the NT disk luminosity goes to zero. 


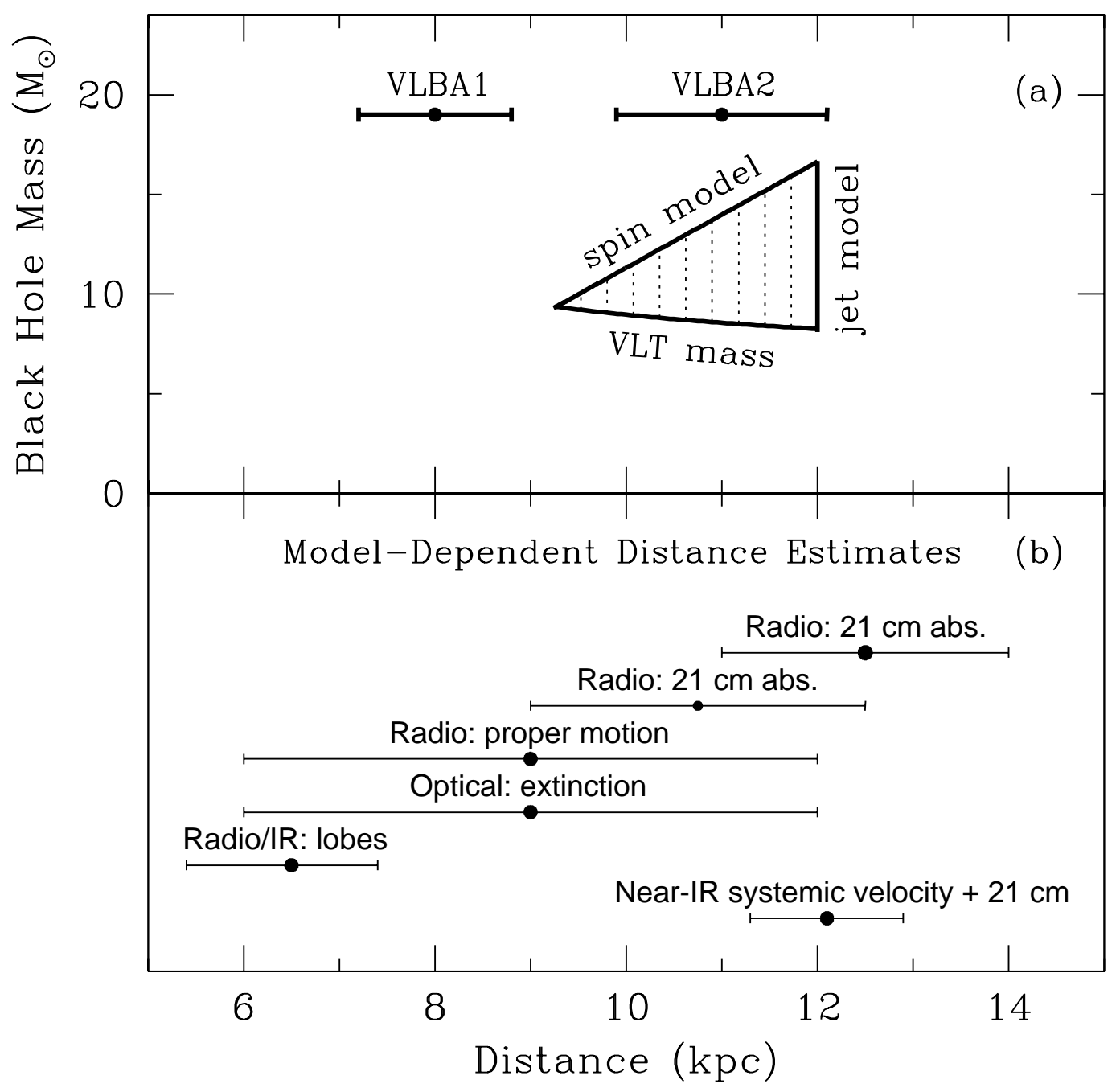

Fig. 5.- (a) Allowed values of black hole mass and distance for GRS 1915+105 fall within the shaded triangular region (see text). (b) Six estimates of the distance to GRS 1915+105 are shown. They range from below 7 to above $12 \mathrm{kpc}$. We are working toward a $10 \%$ trigonometric distance. Two hypothetical and possible outcomes of our VLBA observations, labeled VLBA1 and VLBA2, are indicated at the top of panel $a$. For references on distance estimates, see Figure 18 in McClintock et al. (2006). 

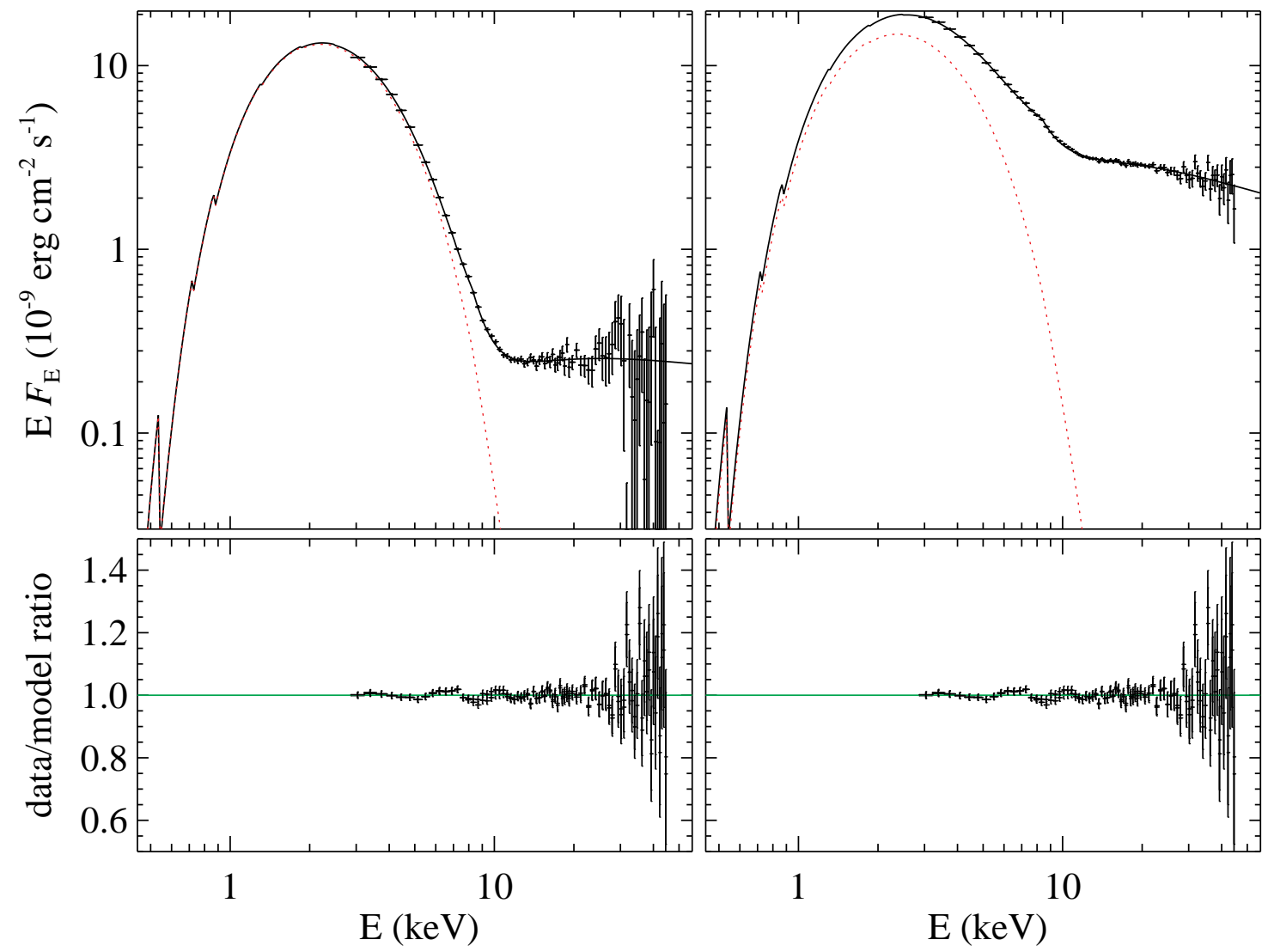

Fig. 6.- Model fits for a pair of spectra of XTE J1550-564. (left) A spectrum with a strongly dominant thermal component; shown are the data, the fit to the data and the fitted thermal component. (right) A strongly Comptonized spectrum. Note the intensity of the power-law component relative to its intensity in the left panel. For details, see Figure 4 and text in Steiner et al. (2010b). 